\title{
Polarization Effects in Relativistic Nuclear Pairing
}

\author{
E.F. Batista, B.V. Carlson, C. De Conti, and T. Frederico \\ Departamento de Física, ITA-CTA, São José dos Campos, SP, Brasil
}

Received on 30 October, 2002

\begin{abstract}
In the present work we discuss the effects of the medium polarization on the nuclear pairing, in the context of the relativistic Hartree-Fock-Bogoliubov (HFB) approximation. The medium polarization effects on the scalar and vector masses, as well on the pairing, as a function of Fermi momentum, are shown for several values of the coupling constants scaled by a parameter $\mathrm{x}$. We find have obtained that the nuclear pairing is strongly affected by the medium polarization.
\end{abstract}

For nearly three decades the nuclear pairing model has played an important role in the description of nuclear spectra. The mean field BCS method has been the most used approximation to solve this problem. However, the BCS theory is a very simple approximation which neglects any contribution to the nucleon-nucleon interaction beyond the bare potential. In order to account for many nuclear properties it is necessary to go beyond the pure BCS approach and add other effects to the nucleon-nucleon interaction, such as the medium polarization and other corrections to the selfenergy.

In models of pairing, one of the most important quantities to be evaluated is the gap equation. In the BCS approach it is possible to obtain an analytical solution for the gap equation as opposed to potential models where this is possible only with a numerical treatment. However, going consistently beyond the BCS approximation including the medium polarization is a difficult task. Analytical results can be obtained only at low density where it has been noted that the medium effect modifies the effective nucleonnucleon interaction by adding a repulsive term, thus causing a reduction in the ${ }^{1} S_{0}$ gap.

Understanding the role played by the nuclear medium in modifying hadronic properties is one of the most interesting problems facing nuclear physics today. One of the most interesting effects of the nuclear medium is the mixing of vector and scalar mesons. In lowest order this mixing is generated by the coupling of the mesons to particle-hole excitations. In the isoscalar channel, the coupling of the $\sigma$ and $\omega$ fields through particle-hole excitations is extremely large and is therefore expected to strongly affect the propagation of mesons in nuclear matter [1].

In this work, we intend to use the effective nucleonnucleon interaction in the ${ }^{1} S_{0}$ pairing channel to study the effect of the medium-modified meson masses. We use a relativistic Hartree-Fock-Bogoliubov formulation, developed in Ref. [2], in which, the QHD formulation is used to describe the nucleon-nucleon interaction in terms of the self-energy, $\Sigma$, and the pairing field, $\Delta$. This model is an improvement over the non-relativistic formulation because it allows a si- multaneous description of many nuclear matter properties such as the saturation point, the effective mass of the nucleon and the pairing energy.

Using a Dirac-HFB approximation to pairing in symmetric nuclear matter, the self-consistency equation for the selfenergy and pairing field are written respectively as [2]

$$
\begin{aligned}
\Sigma(k) & =-i \Gamma_{a}(0) D^{a b}(0) \int \frac{d^{4} q}{(2 \pi)^{4}} \operatorname{Tr}\left[\Gamma_{b}(0) G(q)\right] \\
& +i \int \frac{d^{4} q}{(2 \pi)^{4}} \Gamma_{a}(q) D^{a b}(k-q) G(q) \Gamma_{b}(q)
\end{aligned}
$$

and

$$
\Delta(k)=\int \frac{d^{4} q}{(2 \pi)^{4}} \Gamma_{a}(q) D^{a b}(k-q) F(q) B \Gamma_{b}^{T}(-q) B^{\dagger}
$$

where, $\Gamma_{j \alpha}$ represents the meson-baryon vertex ( we will consider only the scalar and vector vertices), $D_{j}^{\alpha \beta}(k-q)$ is the meson propagator, the matrix B relates transposed quantities to the complex conjugates of the time-reversed ones. It is given by $B=\tau_{2} \otimes \gamma_{5} C$, where $\mathrm{C}$ is the charge conjugation and $\tau_{2}$ is a Pauli matrix that acts in the isospin space. $F(q)$ is the anomalous propagator that is one of the components of the baryon propagator $S_{F}(q)$, which, in the Dirac-HFB formalism, takes the form shown in Fig. 1.

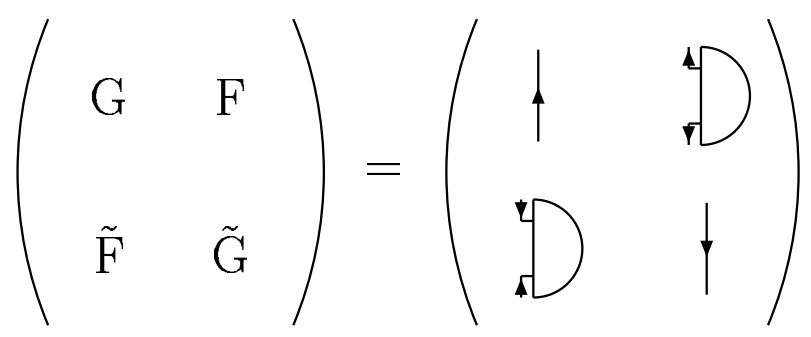

Figure 1. A diagrammatic representation of the components of the HFB propagator. 
in which $\mathrm{G}$ is the usual baryon propagator and $\tilde{F}$ and $\tilde{G}$ are the corresponding time-reversed propagators. The Dyson equation for the usual baryon propagator is given by,

$$
G(k)=G^{0}(k)+G^{0}(k) \Sigma(k) G(k) .
$$

The diagramatic representation for the baryon propagator in a Hartee-Fock approximation is shown in Fig. 2 where the double line denotes the full propagator and the single line denotes the free propagator.

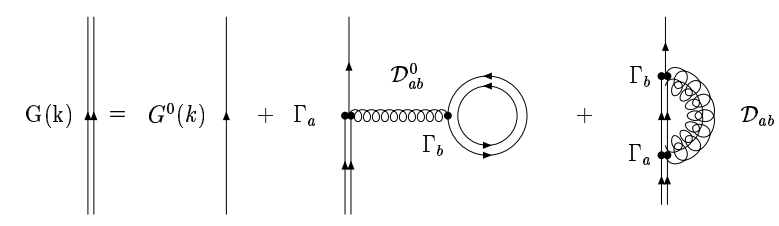

Figure 2. Baryon propagator in HF approximation.

To compute the meson propagator, we sum over ring diagrams, which consist of repeated insertions of the lowestorder one-loop proper polarization part. The sum, which is shown in Fig. 3, yields the relativistic random-phase approximation(RRPA),

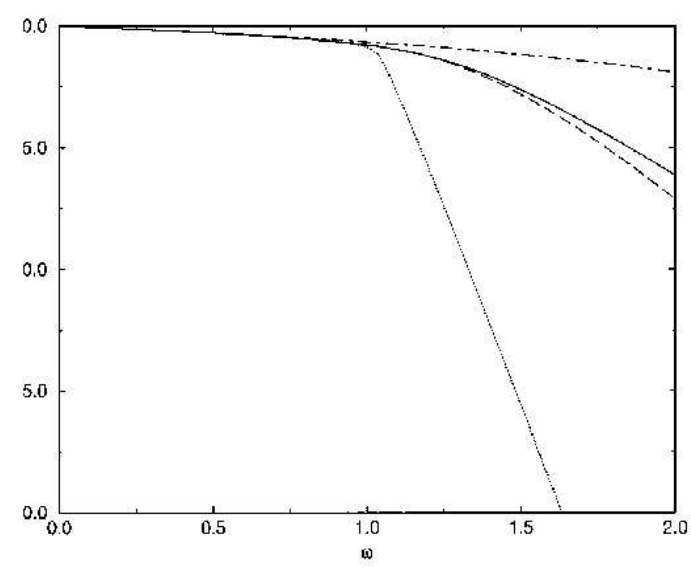

Figure 3. Random-phase approximation for the effective interaction.

The Dyson's equation for the full propagator, $\mathcal{D}$, is given in a matrix form as,

$$
\mathcal{D}_{a d}=\mathcal{D}_{a d}^{0}+\mathcal{D}_{a b}^{0} \Pi_{b c} \mathcal{D}_{c d}
$$

where $\mathcal{D}^{0}$ is the lowest order meson propagator and $\Pi$ is the polarization tensor. Since we want to include $\sigma-\omega$ mixing, it is convenient to use a meson propagator in the form of a 5 $\mathrm{X} 5$ matrix. The lowest order meson propagator is given by a block-diagonal matrix as,

$$
\mathcal{D}^{0 a}{ }_{b}=\left(\begin{array}{cc}
D^{0 \mu} & 0 \\
0 & \Delta^{0}
\end{array}\right) .
$$

The noninteracting propagators for the $\sigma$ and $\omega$ mesons are given respectively by,

$$
\begin{aligned}
\Delta^{0}(q) & =\frac{1}{q_{\mu}^{2}-m_{s}^{2}+i \varepsilon} \\
D_{\mu \nu}^{0}(q) & =\frac{-g_{\mu \nu}}{q_{\mu}^{2}-m_{v}^{2}+i \varepsilon} .
\end{aligned}
$$

The polarization tensor is obtained from the ring diagram, shown in Fig. 4

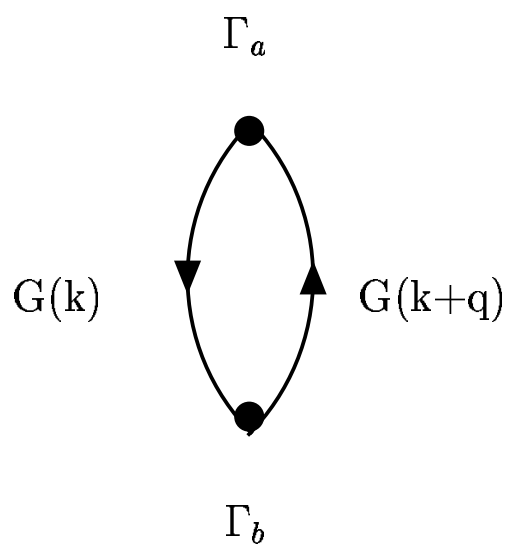

Figure 4. The lowest-order polarization diagram.

where the $\Gamma$ 's are the vertex function. The polarization insertion is given by,

$$
\Pi_{a b}(q)=\left(\begin{array}{cc}
\Pi^{\mu} & \Pi^{m}{ }_{\nu} \\
\Pi^{\mu}{ }_{m} & \Pi_{s}
\end{array}\right) .
$$

The polarizations $\Pi_{\nu}^{\mu}$ and $\Pi^{\mu}$ satisfy current conservation conditions,

$$
\begin{aligned}
q_{\mu} \Pi_{\nu}^{\mu} & =\Pi_{\nu}^{\mu} q^{\nu}=0 \\
q_{\mu} \Pi_{m}^{\mu} & =0 .
\end{aligned}
$$

The lowest order scalar, vector and scalar-vector-mixed polarization are given respectively as

$$
\begin{aligned}
\Pi_{s}(q) & =-i g_{s}^{2} \int \frac{d^{4} k}{(2 \pi)^{4}} \operatorname{Tr}[G(k) G(k+q)], \\
\Pi_{\nu}^{\mu}(q) & =-i g_{s}^{2} \int \frac{d^{4} k}{(2 \pi)^{4}} \operatorname{Tr}\left[\gamma^{\mu} G(k) \gamma_{\nu} G(k+q)\right], \\
\Pi_{\mu}^{M}(q) & \left.=-i g_{s} g_{v} \int \frac{d^{4} k}{(2 \pi)^{4}} \operatorname{Tr}\left[\gamma_{\mu} G(k) G(k+q)\right] 10\right)
\end{aligned}
$$

The nucleon propagator, G(q), is written as a sum of Feynman and density-dependent contributions, 


$$
\begin{aligned}
G(k) & =G^{F}(k)+G^{D}(k), \\
G^{F}(k) & =\left(\gamma^{\mu} k_{\mu}^{\star}+M^{\star}\right) \frac{1}{k_{\mu}^{\star 2}-M^{\star 2}+i \varepsilon}, \\
G^{D}(k) & =\left(\gamma^{\mu} k_{\mu}^{\star}+M^{\star}\right) \frac{i \pi}{E_{k}^{\star}} \delta\left(k_{0}^{\star}-E_{k}^{\star}\right) \theta\left(k_{F}-|\vec{k}| 1,1\right)
\end{aligned}
$$

where $k_{\mu}^{\star}=\left(k_{0}-g_{v} V^{0}, \vec{k}\right)$ and $E_{k}^{\star}=\sqrt{\vec{k}^{2}+M^{\star 2}}$.

With this form of the baryon propagator, each polarization insertion can be divided into two pieces: the Feynman part or vacuum polarization that does not involve the deltafunction and the delta-function density-dependent part. In this work we are not interested in the vacuum contribution and thus will disconsider the term containing only vacuum contributions. We will consider only the density-dependent part of the polarization insertion that also contains a vacuum contribution.

For symmetry of the integrals given by Eq.[10] and current conservation, the only nonnull conponents, at low energy and neglecting recoil, are

$$
\begin{gathered}
\Pi_{S}\left(k_{f}, M^{\star}\right)=\frac{g_{s}^{2}}{2 \pi^{2}}\left[k_{f} E_{f}+3 M^{\star 2} \ln \frac{M^{\star}}{E_{f}+k_{f}}\right], \\
\Pi_{L}\left(k_{f}, M^{\star}\right)=-\frac{g_{v}^{2}}{\pi^{2}} k_{f} E_{f}, \\
\Pi_{M}\left(k_{f}, M^{\star}\right)=\frac{g_{s} g_{v}}{\pi^{2}} k_{f} M^{\star}, \\
\Pi_{T}\left(k_{f}, M^{\star}\right)=-\frac{g_{v}^{2}}{\pi^{2}}\left[k_{f} E_{f}+M^{\star 2} \ln \frac{M^{\star}}{E_{f}+k_{f}}\right],
\end{gathered}
$$

where, $\Pi_{S}, \Pi_{L}, \Pi_{M}, \Pi_{T}$ are the scalar, longitudinal, mixed and transversal polarizations respectivily, with

$$
\Pi_{L}=-\left(\Pi_{3}^{3}+\Pi_{4}^{4}\right) \quad \text { e } \quad \Pi_{M}=\frac{\sqrt{-q^{2}}}{q_{3}} \Pi^{4} .
$$

The sum over ring diagrams to all orders produces a extremely strong polarizability and an instability of nuclear matter. To reduce this effect we are introduce a free parameter $\mathrm{x}$, which scales the coupling constants in the integrals. In Fig. [5] we plot the polarizations as a function of the Fermi momentum for $x=0.1$, in which, we show the density-dependence of the polarization. We note the strong sensitivity of polarizations with the density.

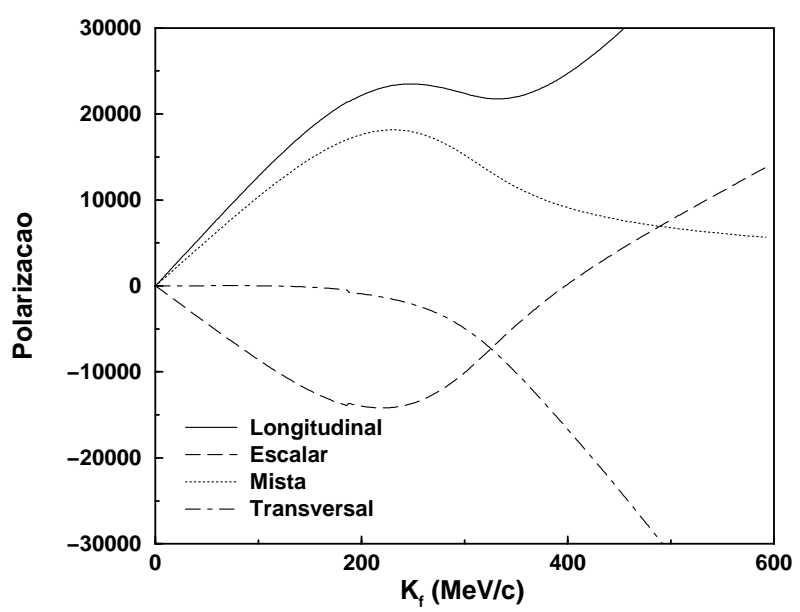

Figure 5. Polarization as a function of the fermi momentum.

The effective mass of the mesons in nuclear matter is obtained by diagonalizing the meson propagator,

$$
\left(\mathcal{D}^{-1}\right)^{a}{ }_{b}=\left(q^{2}-m^{2}\right) \delta_{b}^{a}-\Pi^{a}{ }_{b} .
$$

After obtaining the eigenvectors, the meson propagators can be written in the form,

$$
\mathcal{D}^{a b}=\sum_{i} u_{i}^{a} D_{i} u_{i}^{b}=\sum_{i} u_{i}^{a} \frac{1}{q^{2}-m_{i}^{2}} u_{i}^{b}
$$

where $u_{i}^{a}$ are the eigenvectors of meson propagator.

In the Figs. 6, 7, 8 we show the modified meson masses as a function of the Fermi momentum for several values of the free parameter $\mathrm{x}$. We note that the polarization contribution increases rapidly with the free parameter $\mathrm{x}$. The polarization contributions decrease the scalar meson mass and increase the vector meson mass. Their combined effect is to make the effective nucleon-nucleon interation more atractive.

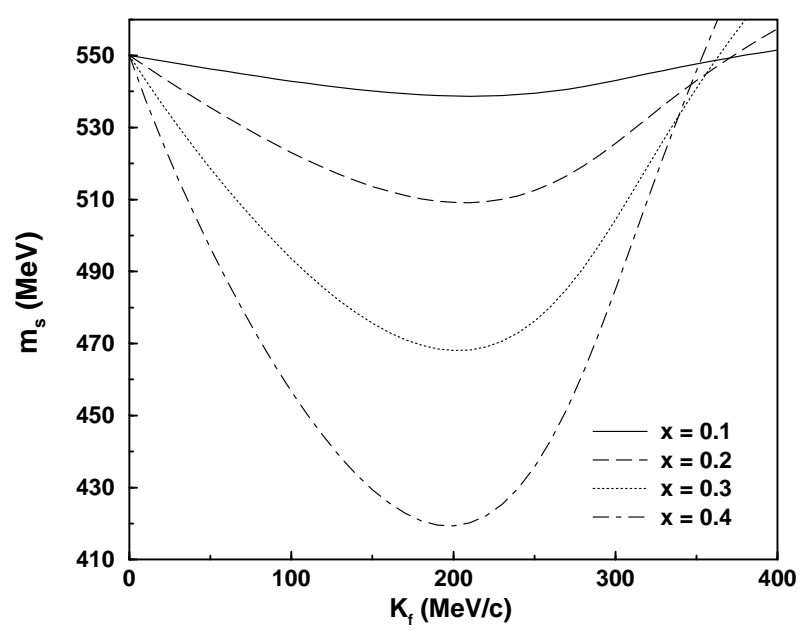

Figure 6. Scalar meson mass. 


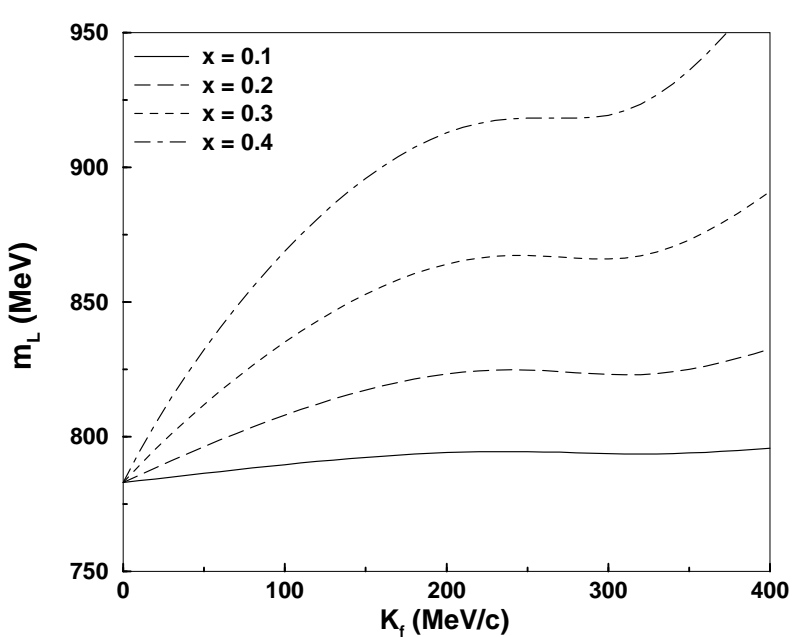

Figure 7. Longitudinal vector meson mass.

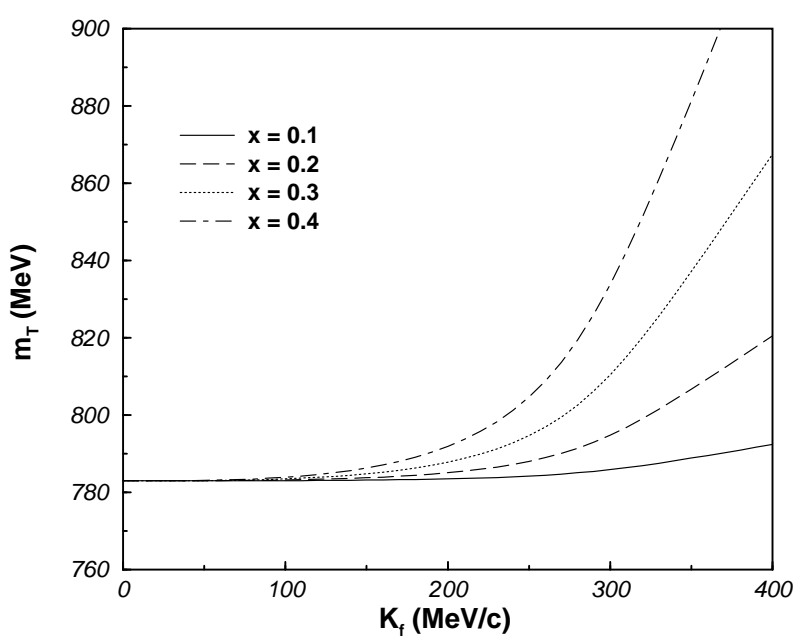

Figure 8. Transversal vector meson mass.

The Hermiticity and transposition invariance conditions, as well as the requirements of invariance under Lorentz and parity transformation reduce the possible form of the selfenergy, in symmetric nuclear matter, to

$$
\Sigma(k)=\Sigma_{S}(k)-\gamma_{0} \Sigma_{0}(k)+\vec{\gamma} \cdot \vec{k} \Sigma_{T}(k) ;
$$

while, the pairing field can be taken to be,

$$
\Delta(k)=\left[\Delta_{S}(k)-\gamma_{0} \Delta_{0}(k)-i \gamma_{0} \vec{\gamma} \cdot \vec{k} \Delta_{T}(k)\right] \vec{\tau} \cdot \vec{n} .
$$

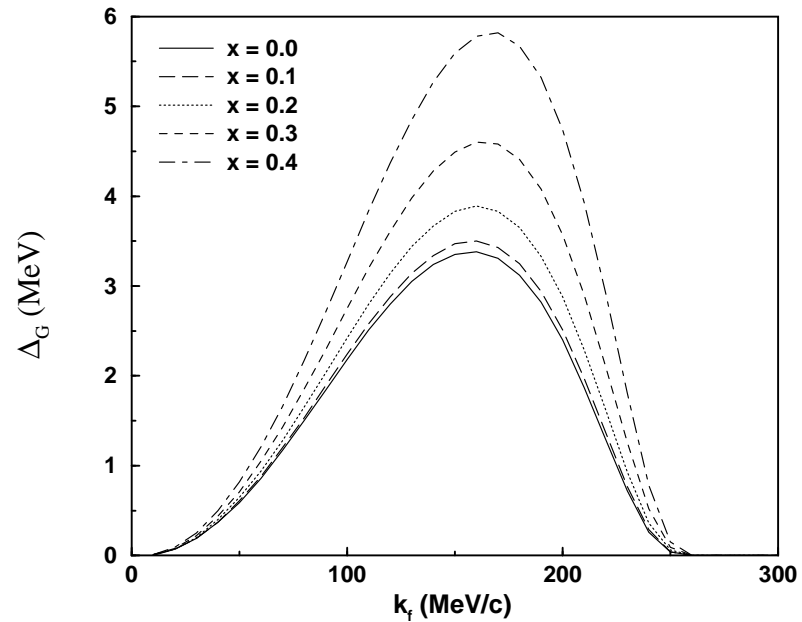

Figure 9. Pairing gap.

In Fig. 9 we plot the pairing gap as a function of Fermi momentum for several values of free parameter. The increasingly attractive effective nucleon-nucleon interaction causes a strong increase on the pairing gap.

We conclude that, the polarization effect is very strong changing drastically the effective nucleon-nucleon interaction. The pairing field is extremely sensitive to the medium effect.

\section{Acknowledgments}

E.F.B acknowledges the support of FAPESP. B.V.C. and T. Frederico acknowledge partial support from FAPESP and CNPq.

\section{References}

[1] L.S. Celenza, A. Pantzires and C.M. Shakin, Phys. Rev. C45, 205 (1992). H.-C.Jean, J. Piekarewicz and G. A. Williams, Phys. Rev. C49, 1981 (1994).

[2] F.B. Guimarães, B.V. Carlson and T. Frederico, Phys. Rev. C54, 2385 (1996); F.B. Guimarães, B.V. Carlson and T. Frederico, Phys. Rev. C56, 3097 (1997).

[3] R. J. Furnsthal and C. J. Horowitz, Nuc. Phys. A485, 632 (1988); K. Lim and C. J. Horowitz Nuc. Phys. A490, 729 (1988); K. Saito, K. Tsushima, A. W. Thomas and A. G. Williams, Phys. Lett. B433, 243 (1998). 